\title{
Small Signal Monitoring of Power System using Subspace System Identification
}

\author{
A. Mohammadi, H. Khaloozadeh, R. Amjadifard
}

\author{
Aliakbar Mohammadi \\ Department of Electrical Engineering \\ Science and Research Branch \\ Islamic Azad University \\ Tehran, Iran. \\ aa.mohammadi@srbiau.ac.ir
}

\section{Hamid Khaloozadeh}

K. N. Toosi University of Technology

Tehran, Iran.

h_khaloozadeh@kntu.ac.ir

\section{Roya Amjadifard}

Tarbiat Moallem University

Tehran, Iran.

amjadifard@tmu.ac.ir

\begin{abstract}
In this paper, small signal analysis of power systems is investigated using Subspace System Identification (SSI) methods. Classical small signal analysis methods for power systems are based on mathematical modeling and linearized model of power system in an especial operating point. There are some difficulties when such a classical method is applied, specially, in the case of large power systems. In this paper, such difficulties and their bases are investigated and in order to avoid them, it is suggested to use SSI algorithms for small signal analysis of power systems. The paper discusses extracting of small signal properties of power systems and presents some new suggestions for application of subspace system identification methods. Different types of subspace system identification algorithms were applied to different power system case studies using the presented propositions. The benefits and drawbacks of subspace system identification methods and the presented suggestions are studied for small signal analysis of power systems and power system monitoring. Several comparisons were investigated using computer simulations. The results express the usefulness and easiness of proposed methods.
\end{abstract}

Keywords: small signal, subspace identification, power system, monitoring.

\section{Introduction}

It is not more than 20 years that a new horizon has been opened in system identification. Subspace System Identification (SSI) has been one of the most attractive methods for system identification since 1990s. There is a large amount of literatures devoted to the algorithms based on subspace system identification [1-3]. Factually, we can present a list of renowned type of SSI algorithms as following; Numerical Subspace State Space System IDentification (N4SID) [4], Multivariable Output Error State Space (MOESP) [5,6], Past Output (PO-MOESP) [7, 8], Canonical Correlation Analysis (CCA) [9], Orthogonal Decomposition (ORT) [2]. Although they are similar in some general aspects, but there are several differences which may distinguish them. Actually, they don't perform quite the same as each other since they practice various 
mathematical tools in different ways. It is not too far to expect fairly different advantages or disadvantages when using SSI algorithms. A useful review of subspace system identification algorithms is available in [10]. Authors in [1] present a unifying theory which may be helpful to understand SSI methods.

During the past years, application of SSI methods is being developed increasingly to different areas of industry and engineering sciences. Maybe the main reason for such a development hides behind the capability and easiness of application of SSI methods for multi-input/multi-output (MIMO) systems. In addition, state space structure of SSI methods is usually considered as an advantage. SSI methods also use robust, fast and consistent mathematical tools for calculations, which provide them with some considerable advantages [2,3].

Numerous investigators have worked on SSI methods and they have used different SSI algorithms for different applications. Moreover, MIMO specifications and also state space based analysis motivated many investigators to apply SSI methods for different analysis of power systems. However, there are not so many SSI applications in power systems, yet. The first steps of SSI applications in power systems may be seen in [11]. The paper provides low order model of large scale power system using N4SID algorithm. Results of paper express that SSI based model is in lower orders, more optimized and more suitable for controller design in comparison with classical system identification and modeling.

An application of power transformer identification was developed in [12] using frequency response data and SSI methods. Authors in [13] and [14] introduce an algorithm based on SSI and some applications of such algorithm for transformer debugging and parameter estimation.

A Heffron-Phillips model of synchronous generator was identified in [15] using subspace identification algorithms and online measurements. In [16] the parameters of a Heffron-Phillips model of synchronous generator were extracted from closed loop data using SSI algorithms. It divides identification problem of a closed loop system to two open loop identification and then it uses SSI algorithms to identify each open loop transfer function. Using some mathematical processing of the provided transfer function, it provides a transfer function as a generator model.

In [17], authors discuss a model predictive controller design for multi-machine power system using SSI algorithms. The design uses a recursive subspace system identification algorithm in order to provide a MIMO self-tuning adaptive controller; therefore it can be used for online applications. [18] mentions use of different types of power system signals which are applicable to SSI algorithms. It uses such signals to provide identification data. Modal analysis of power system was developed using subspace system identification methods and sampled data. [19] also discusses modal analysis and oscillatory stability study of power systems based on SSI methods. It provides a voltage stability measure using identified critical modes of power system. [20] introduces a power system stabilizer (PSS) using N4SID algorithm of SSI methods. It provides a power system model using SSI and then it designs a MIMO power system stabilizer using identified model. In [21], authors also discuss a PSS using stochastic subspace system identification approaches. They also mention small signal analysis of power systems.

This paper aims at small signal analysis of power systems using SSI. It proposes some useful notes on how to extract small signal properties of a power system using SSI algorithms. The paper clarifies the process of small signal monitoring of power system using subspace system identification algorithms. It also compares several SSI based analysis of power systems with the classical methods to distinguish the advantages.

The paper structure is as following; In order to highlight drawbacks of classical methods, there would be an introduction to classical small signal analysis of power systems in the next section. Subspace system identification algorithms are discussed in section three to high-light the advantages of such methods. Application of SSI methods for power system small signal analysis is introduced in section four. There are some new ideas in section four, which are used to extract 
small signal properties of test case power systems in section five. On-line monitoring of power system is discussed in section six. In section seven, the application of different SSI algorithms for several power systems is discussed. Finally, the conclusions are presented.

\section{Drawbacks of Analytically Small Signal Analysis of Power Sys- tems}

We are usually interested in extraction of modes, participation factors of modes, damping ratios and oscillatory frequencies of power system which all are called small signal properties of power systems. Since a power system is naturally a nonlinear system, one should follow the following stages to achieve small signal properties of a power system:

1. Finding the details of all included elements (Generator constants, Transformer and line parameters,)

2. Finding nonlinear model of power system using constant, parameters and theoretical relations of variables for different power system elements.

3. Solving a load flow problem in order to provide an operating point.

4. Linearizing of nonlinear model using the provided operating point.

5. Application of modern small signal methods to provide small signal properties.

Providing an operating point, a nonlinear modeling and linearizing the model are all tough works in application, especially when the system is large. We also know that the parameters of system may change during the normal operation of system. Therefore, it is obvious that some of the above stages are not applicable to a real system or the result of such an analysis is not reliable. Moreover, There is always a big gap between the analysis done on a piece of paper and the system behavior. Such a method is not applicable for monitoring of power system, neither. This is a considerable drawback for a scientific method. It is always a question at the end of theoretical analysis of power systems; To what extent are the results useful and applicable? it is not easy to response to the question unless we make a bridge between real world phenomena and theoretical analysis.

What can be suggested at this point is usually application of a system identification method, at least, for estimating a linear model. Classical identification methods are useful in many applications. While using a classical system identification method, the biggest difficulty origins from single-input/single-output (SISO) structure of such methods. Classical system identification methods may fall into whirlpool of over parameterization. Coping with such problems is itself a new problem.

Our suggestion for overcoming such problems is to use subspace system identification (SSI) methods. SSI methods are good solution for multi-input/multi-output (MIMO) systems. They can be considered as the bridge for passing over the gap between real world system and theoretical analysis. The next section investigates SSI methods to glorify their useful advantages for small signal analysis of power systems.

\section{Subspace System Identification}

Generally, we can arrange SSI methods into two categories from the measurement view; stochastic and deterministic SSI algorithms. If the SSI algorithm uses exogenous input measurements in its raw identification data set, it is called as deterministic SSI algorithm (DSSI). 
Otherwise, it is called a Stochastic Subspace System Identification (SSSI) method [2,22]. If it is supposed to provide SSSI methods in an algorithmic way for further understanding, we can arrange the following notations. Further investigations of the following notes are also useful for understanding of DSSI algorithms;

Model: The considered model of system for a typical SSSI algorithm is

$$
\left\{\begin{array}{l}
x(t+1)=A x(t)+w(t) \\
y(t)=C x(t)+v(t)
\end{array}, E\left\{\left[\begin{array}{c}
w(t) \\
v(t)
\end{array}\right]\left[\begin{array}{ll}
w^{T}(s) & v^{T}(s)
\end{array}\right]\right\}=\left[\begin{array}{cc}
Q & S \\
S^{T} & R
\end{array}\right] \delta_{t s}\right.
$$

where $y \in \mathbb{R}^{n_{y}}, x \in \mathbb{R}^{\mathrm{n}}$ are samples of output and state vectors. $v_{\mathrm{t}} \in \mathbb{R}^{n_{y}}, w_{\mathrm{t}} \in \mathbb{R}^{\mathrm{n}}$ are stationary, zero average state and output noise vectors, consequently.

Identification Data: SSSI methods use only samples of system outputs. However, identification data should usually be provided in the following structure:

$$
\begin{aligned}
& f(t) \triangleq\left[\begin{array}{llll}
y(t) & y(t+1) & \cdots & y(t+k-1)
\end{array}\right]^{T} \\
& p(t) \triangleq\left[\begin{array}{llll}
y(t-1) & y(t-2) & \cdots & y(t-k)
\end{array}\right]^{T}
\end{aligned}
$$

where $f(t)$ and $p(t)$ are future and past data set. $\mathrm{k}$ should be strictly bigger than $\mathrm{n}$. It can be a guess. Therefore, this is not a restrictive condition. Now, we can formulate SSSI problem as below $[1,2,5,10,22]$ :

"There are $N$ samples of output vectors, $Y(t) \triangleq\left[\begin{array}{llll}y(0) & y(1) & \cdots & y(N-1)\end{array}\right]$, from a system of order $n$. Find matrices $A, C, Q, R, S$ and $n$ for the structure defined in (1)."

Block Hankel Matrices: SSSI algorithms begin data processing by forming the following block Hankel Matrices:

$$
H_{k, k}=E\left\{f(t) p^{T}(t)\right\}=\left[\begin{array}{cccc}
\Lambda(1) & \Lambda(2) & \cdots & \Lambda(k) \\
\Lambda(2) & \Lambda(3) & \cdots & \Lambda(k+1) \\
\vdots & \vdots & \ddots & \vdots \\
\Lambda(k) & \Lambda(k+1) & \cdots & \Lambda(2 k-1)
\end{array}\right]
$$

where $\Lambda(l)=E\left\{y(t+l) y^{T}(l)\right\}, \quad l=0,1, \cdots, L, \quad 2 k-1 \leq L, \quad k>n$ is the correlation of future and past data. Actually, SSSI uses statistical properties of output samples for further processing. The word Stochastic in the expression (Stochastic Subspace System Identification) may arise from this point.

System Order: SSSI uses the following Singular Value Decomposition (SVD) of Hankel matrix in order to provide system order $(n)$ :

$$
H_{k, k}=\left[\begin{array}{ll}
U_{\text {sys }} & U_{\text {noise }}
\end{array}\right]\left[\begin{array}{cc}
\sum_{\text {sys }} & 0 \\
0 & \sum_{\text {noise }}
\end{array}\right]\left[\begin{array}{c}
V_{\text {sys }}^{T} \\
V_{\text {noise }}^{T}
\end{array}\right] \simeq U_{\text {sys }} \Sigma_{\text {sys }} V_{\text {sys }}^{T}
$$

We can detect noise singular values by detecting a big gap among the singular values of Hankel matrix. Thus, noise singular values can be neglected since they are very smaller than system singular values. Therefore, system order, $n$ can be defined as

$$
n \triangleq \operatorname{dim}\left(\Sigma_{\text {sys }}\right)
$$

Estimation of State Space Matrices: $S V D$ of Hankel matrix can also provide us with an extended observability matrix;

$$
O_{k} \triangleq U_{s y s} \Sigma_{s y s}^{1 / 2}
$$


It can also present controllability matrix:

$$
C_{k} \triangleq \Sigma_{\text {sys }}^{1 / 2} V_{\text {sys }}^{T}
$$

We can easily obtain system matrix using extended observability matrices:

$$
\begin{aligned}
& A=O_{k-1}^{\dagger} O_{k}\left(p+1: k n_{y}, 1: n\right) \\
& C=O_{k}\left(1: n_{y}, 1: n\right)
\end{aligned}
$$

Estimation of Variance Matrices: If we define

$$
\bar{C}^{T}=C_{k}\left(1: n, 1: n_{y}\right)
$$

Variance matrices can be evaluated as below;

$$
\begin{aligned}
& Q=\Pi_{*}-A \Pi A^{T}, \\
& S=\bar{C}^{T}-A \Pi_{*} C^{T}, \\
& R=\Lambda(0)-C \Pi_{*} C^{T}
\end{aligned}
$$

where the essentials of the above formulation is provided by solving the following algebraic Riccati equation;

$$
\begin{aligned}
& \Pi_{k+1}=A \Pi_{k} A^{T}+\left(\bar{C}^{T}-A \Pi_{k} C^{T}\right)\left(\Lambda(0)-C \Pi_{k} C^{T}\right)^{-1}\left(\bar{C}-C \Pi_{k} C^{T}\right) \\
& \Pi_{*}=\lim _{k \rightarrow \infty} \Pi_{k}
\end{aligned}
$$

Innovation Model for state estimation: If we are interested in innovation model for state estimation, we should have an estimation of Kalman gain matrix:

$$
K=\left(\bar{C}^{T}-A \Pi_{*} C^{T}\right)\left(\Lambda(0)-C \Pi_{*} C^{T}\right)^{-1}
$$

Therefore, states can be estimated using the following dynamic equations,

$$
\begin{aligned}
& x(t+1)=A x(t)+K e(t) \\
& y(t)=C x(t)+e(t) \\
& \operatorname{cov}\{e(t)\}=R
\end{aligned}
$$

Considering above mentioned notations, we can investigate the deterministic version of SSI algorithms using the following model:

$$
\left\{\begin{array}{l}
x(t+1)=A x(t)+B u(t)+w(t) \\
y(t)=C x(t)+D u(t)+v(t)
\end{array}, E\left\{\left[\begin{array}{c}
w(t) \\
v(t)
\end{array}\right]\left[\begin{array}{ll}
w^{T}(s) & v^{T}(s)
\end{array}\right]\right\}=\left[\begin{array}{cc}
Q & S \\
S^{T} & R
\end{array}\right] \delta_{t s}\right.
$$

where $u_{\mathrm{t}} \in \mathbb{R}^{n_{u}}, y_{\mathrm{t}} \in \mathbb{R}^{n_{y}}, x_{\mathrm{t}} \in \mathbb{R}^{\mathrm{n}}$ are samples of input, output, state vectors and $v_{\mathrm{t}} \in \mathbb{R}^{n_{y}}, w_{\mathrm{t}} \in$ $\mathbb{R}^{\mathrm{n}}$ are stationary, zero mean state noise and output noise vectors. In the case of deterministic identification, subspace system identification problem can be formulated as below $[2,4,22]$ : "There are $N$ samples of input vectors $u=\left[\begin{array}{lllll}u_{0} & u_{1} & u_{2} & \ldots & u_{N-1}\end{array}\right]$ and output vectors $y=$ $\left[\begin{array}{lllll}y_{0} & y_{1} & y_{2} & \ldots & y_{N-1}\end{array}\right]$ from a system of order $n$. Find $A, B, C, D, Q, R, S$ matrices and $n$ for the structure defined in (14)."

There are several different algorithms available for SSI. They usually use some consistence mathematical tools that provide them with pretty useful benefits. There are two well-known subspace system identification algorithms expressed in Table 1. They use the same measurements, the same block Hankel matrices, different types of projections, SVD of different matrices, 
the same method for extraction of system order and different extended observability matrices. MOESP does not need to estimate future states of system, but N4SID provides future state vectors by using a weighting matrix. MOESP uses extended observability matrix to extract system matrices but N4SID uses future states and through a least square problem estimates the system matrices. Investigating Table 1 expresses the following advantages for subspace system identification algorithms:

1. SSI Algorithms are the only system identification methods that can easily and extensively be applied to all MIMO and SISO systems.

2. Estimation of system order is one of the steps of SSI algorithms. This advantage reduces amount of time, cost and calculations.

3. SSI methods can handle big packages of data.

4. On-line operations of SSI methods are easier and can easily be applied to MIMO systems.

5. SSI methods use robust mathematical tools such as SVD, LQ decomposition, least square and QR decomposition. They also don't need nonlinear optimization.

6. Some SSI algorithms only use output data to identify a model. This is a considerable advantage.

\section{Application of SSI Methods for Small Signal Analysis of Power Systems}

The SSI advantages expressed in previous section can be used to overcome the difficulties with classical small signal analysis of power systems. Using SSI methods reduces above five steps to the following three steps:

1. Measuring input/output signals of power system.

2. Identification of a linear model for power system using SSI algorithms.

3. Application of modern small signal methods to provide small signal properties.

As it can be seen, the four first steps vanished and two other steps replaced them. The fifth step left with no change. Therefore, one can provide small signal analysis of power systems in an easier and faster way. Since application of SSI algorithms are very easy, power system small signal analysis will be provided in very low levels of cost and time.

We are usually interested in identification of most oscillatory and least damped modes of power system. Such modes are usually related to electro-mechanical parts of power system. Therefore, in order to identify most critical modes, the angle and speed of electrical machines should be measured. Signal measuring is the starting point of system identification. Since measured signals should have enough persistency of excitation, we should use most effective inputs. In attention to differential equations of a single machine power system [23], mechanical torque and field voltage are proper inputs.

Suppose that input vector $u$ and output vector $y$ of a power system have been measured. The goal is to find small signal properties of power system (Modes, Damping Ratios, Oscillation Frequencies, Participation Factors) using several samples of $u$ and $y$. It is announced that if 


\begin{tabular}{|c|c|c|c|}
\hline step & Operation & MOESP Algorithm & N4SID Algorithm \\
\hline 1 & Model & $\begin{array}{l}\left\{\begin{array}{l}x_{t+1}=A x_{t}+B u_{t}+w_{t} \\
y_{t}=C x_{t}+D u_{t}+v_{t}\end{array}\right. \\
E\left\{\left[\begin{array}{c}w_{t} \\
v_{t}\end{array}\right]\left[\begin{array}{ll}w_{s}^{T} & v_{s}^{T}\end{array}\right]\right\} \\
=\left[\begin{array}{cc}Q & S \\
S^{T} & R\end{array}\right] \delta_{t s}\end{array}$ & $\begin{array}{l}\left\{\begin{array}{l}x_{t+1}=A x_{t}+B u_{t}+w_{t} \\
y_{t}=C x_{t}+D u_{t}+v_{t}\end{array}\right. \\
E\left\{\left[\begin{array}{c}w_{t} \\
v_{t}\end{array}\right]\left[\begin{array}{ll}w_{s}^{T} & v_{s}^{T}\end{array}\right]\right\} \\
=\left[\begin{array}{cc}Q & S \\
S^{T} & R\end{array}\right] \delta_{t s}\end{array}$ \\
\hline 2 & Measured Data & $\begin{aligned} u & =\left[\begin{array}{lllll}u_{0} & u_{1} & u_{2} & \ldots & u_{N-1}\end{array}\right] \\
y & =\left[\begin{array}{lllll}y_{0} & y_{1} & y_{2} & \ldots & y_{N-1}\end{array}\right]\end{aligned}$ & $\begin{array}{l}u=\left[\begin{array}{lllll}u_{0} & u_{1} & u_{2} & \ldots & u_{N-1}\end{array}\right] \\
y=\left[\begin{array}{lllll}y_{0} & y_{1} & y_{2} & \ldots & y_{N-1}\end{array}\right]\end{array}$ \\
\hline 3 & $\begin{array}{l}\text { Block Hankel } \\
\text { Matrices }\end{array}$ & $\begin{array}{l}U_{0, k-1}= \\
{\left[\begin{array}{cccc}u_{0} & u_{1} & \cdots & u_{N-1} \\
u_{1} & u_{2} & \cdots & u_{N} \\
\vdots & \vdots & \vdots & \vdots \\
u_{k-1} & u_{k} & \cdots & u_{k+N-2}\end{array}\right]} \\
\in \mathbb{R}^{k n_{u} \times N} \\
Y_{0, k-1}= \\
{\left[\begin{array}{cccc}y_{0} & y_{1} & \cdots & y_{N-1} \\
y_{1} & y_{2} & \cdots & y_{N} \\
\vdots & \vdots & \vdots & \vdots \\
y_{k-1} & y_{k} & \cdots & y_{k+N-2} \\
\in \mathbb{R}^{k n_{y} \times N} & & \end{array}\right.}\end{array}$ & $\begin{array}{l}U_{0, k-1}= \\
{\left[\begin{array}{cccc}u_{0} & u_{1} & \cdots & u_{N-1} \\
u_{1} & u_{2} & \cdots & u_{N} \\
\vdots & \vdots & \vdots & \vdots \\
u_{k-1} & u_{k} & \cdots & u_{k+N-2}\end{array}\right]} \\
\in \mathbb{R}^{k n_{u} \times N} \\
Y_{0, k-1}= \\
{\left[\begin{array}{cccc}y_{0} & y_{1} & \cdots & y_{N-1} \\
y_{1} & y_{2} & \cdots & y_{N} \\
\vdots & \vdots & \vdots & \vdots \\
y_{k-1} & y_{k} & \cdots & y_{k+N-2} \\
\in \mathbb{R}^{k n_{y} \times N} & \end{array}\right.}\end{array}$ \\
\hline 4 & $\begin{array}{l}\text { Extra Prede- } \\
\text { fined Matrices }\end{array}$ & $\begin{array}{l}U_{p} \triangleq U_{0, k-1} \\
Y_{p} \triangleq Y_{0, k-1}\end{array}$ & $\begin{array}{l}U_{p} \triangleq U_{0, k-1}, \quad Y_{p} \triangleq Y_{0, k-1} \\
U_{f} \triangleq U_{k, 2 k-1}, Y_{f} \triangleq Y_{k, 2 k-1} \\
W_{p} \triangleq\left[\begin{array}{c}U_{p} \\
Y_{p}\end{array}\right], \quad W_{f} \triangleq\left[\begin{array}{c}U_{f} \\
Y_{f}\end{array}\right.\end{array}$ \\
\hline 5 & $\begin{array}{l}\text { LQ Decomposi- } \\
\text { tion }\end{array}$ & $\begin{array}{l}{\left[\begin{array}{l}U_{p} \\
Y_{p}\end{array}\right]} \\
{\left[\begin{array}{cc}L_{11} & 0 \\
L_{21} & L_{22}\end{array}\right]\left[\begin{array}{l}Q_{1}^{T} \\
Q_{2}^{T}\end{array}\right]}\end{array}$ & {$\left[\begin{array}{c}U_{f} \\
W_{p} \\
Y_{f}\end{array}\right]$} \\
\hline 6 & Projection & $Y_{p} / U_{p}^{\perp}=L_{22} Q_{2}^{T}$ & $Y_{f} / U_{f} W_{p}=L_{32} L_{22}^{\dagger} W_{p}$ \\
\hline 7 & $\begin{array}{l}\text { Singular Value } \\
\text { Decomposition } \\
\text { (SVD) }\end{array}$ & $\begin{array}{l}L_{22} \\
{\left[\begin{array}{ll}U_{1} & U_{2}\end{array}\right]\left[\begin{array}{cc}\sum_{1} & 0 \\
0 & 0\end{array}\right]\left[\begin{array}{c}V_{1}^{T} \\
V_{2}^{T}\end{array}\right]}\end{array}$ & $\begin{array}{l}L_{32} L_{22}^{\dagger} W_{p} \\
{\left[\begin{array}{ll}U_{1} & U_{2}\end{array}\right]\left[\begin{array}{cc}\sum_{1} & 0 \\
0 & 0\end{array}\right]\left[\begin{array}{c}V_{1}^{T} \\
V_{2}^{T}\end{array}\right]}\end{array}$ \\
\hline 8 & System Order & $n \triangleq \operatorname{dim}\left(\Sigma_{1}\right)$ & $n \triangleq \operatorname{dim}\left(\Sigma_{1}\right)$ \\
\hline 9 & $\begin{array}{ll}\text { Extended } & \text { Ob- } \\
\text { servability } & \\
\text { Matrix } & \end{array}$ & $O_{k} \triangleq U_{1} \sum_{1}^{1 / 2}$ & $O_{k} \triangleq U_{1} \sum_{1}^{1 / 2} T \quad, \quad|T| \neq 0$ \\
\hline 10 & $\begin{array}{l}\text { Future State Es- } \\
\text { timation }\end{array}$ & -—- & $\begin{aligned} X_{f} & =T^{-1} \Sigma_{1}^{1 / 2} V_{1}^{T} \in \mathbb{R}^{n \times N} \\
X_{k} & \triangleq X_{f} \\
& =\left[\begin{array}{llll}x_{k} & x_{k+1} & \cdots & x_{k+N-1}\end{array}\right] \\
& \in \mathbb{R}^{n \times N}\end{aligned}$ \\
\hline 11 & $\begin{array}{l}\text { Estimation of } \\
\text { State Space } \\
\text { Matrixes }\end{array}$ & $\begin{array}{l}\quad C=O_{k}\left(1: n_{y}, 1: n\right) \\
\quad A=O_{k}^{\dagger}\left(1: n_{y}(k-1), 1: n\right) \\
\quad . O_{k}\left(n_{y}+1: k n_{y}, 1: n\right) \\
\text { Solving a Least Equare Problem } \\
\text { to Estimate B and D }\end{array}$ & $\begin{array}{l}{\left[\begin{array}{ll}\hat{A} & \hat{B} \\
\hat{C} & \hat{D}\end{array}\right]=} \\
\left(\left[\begin{array}{c}X_{k+1} \\
Y_{k, k}\end{array}\right]\left[\begin{array}{c}X_{k} \\
U_{k, k}\end{array}\right]^{T}\right) \\
\cdot\left(\left[\begin{array}{c}X_{k} \\
U_{k, k}\end{array}\right]\left[\begin{array}{c}X_{k} \\
U_{k, k}\end{array}\right]^{T}\right)\end{array}$ \\
\hline
\end{tabular}


$N$ samples of input/output vectors are availabe, then one can identify the following state space linear model by utilizing a subspace system system identification algorithm:

$$
x(t+1)=A x(t)+B u(t), \quad y(t)=C x(t)+D u(t)
$$

One can find the system modes and as a result damping factors and damping frequencies by digging the matrix $A$. However, we may encounter some difficulties when calculating the participation factors of modes in states. The problem arises since the state vector $x$ of the identified model is not correspondent to that of the real power system which we may obtain by analytical methods. Therefore, mode in state participation factors can't be utilized using the identified $A$.

In order to cope with such a problem, it is proposed to use modal canonical realization of (15). Using $T$ as a similarity transformation matrix, one can provide the following modal canonical realization:

$$
\begin{gathered}
\dot{z}=\Lambda z+\bar{B} u, \quad y=\bar{C} z+\bar{D} u \\
x=T z, \Lambda=T^{-1} A T, \quad \bar{B}=T^{-1} B, \bar{C}=C T, \quad \bar{D}=D
\end{gathered}
$$

Generally, $\Lambda$ is in Jordan and block diagonal structure. Thus, mode in state participation factor $\left(p_{k i}\right)$ is defined as

$$
p_{k i} \triangleq \frac{\partial \lambda_{i}}{\partial a_{k k}}
$$

where $a_{k k}$ is a diagonal element of system matrix. Since in (16), the system matrix is diagonal with modes as its diagonal elements, we can write:

$$
p_{k i} \triangleq \frac{\partial \lambda_{i}}{\partial a_{k k}}=\frac{\partial \lambda_{i}}{\partial \lambda_{i}}=1
$$

Therefore, modal canonical realization can maximize $(100 \%)$ mode in state participation factor of model. In order to clarify the point, suppose that $u$ is zero, $\Lambda$ is diagonal and $z^{0}$ is the initial condition vector of modal canonical realization. Thus, we can write:

$$
\dot{z}=\Lambda z \quad \Rightarrow \quad z_{i}=e^{-\lambda_{i} t} z_{i}^{0} \quad, i=1,2, \cdots, n
$$

Therefore, the only participating mode in state $z_{i}$ is $\lambda_{i}$, so the participation factor of mode $\lambda_{i}$ in the state $z_{i}$ is $100 \%$ and each mode is mapped to a state. Considering above point and output equation of (15), one can write:

$$
y=\bar{C} z \Rightarrow y_{k}=\sum_{i=1}^{n} \bar{c}_{k i} z_{i}=\sum_{i=1}^{n} \bar{c}_{k i} z_{i}^{0} e^{-\lambda_{i} t}, \quad k=1, \cdots, n_{y}
$$

Therefore, output $y_{k}$ is affected by mode $\lambda_{i}$ and mode in output participation factor $\left(p_{k i}\right)$ is proposed as:

$$
p_{k i} \triangleq \bar{c}_{k i} z_{i}^{0}, k=1, \cdots, n_{y} \text { and } i=1, \cdots, n
$$

In order to provide participation factors, one may need $z^{0}$ which can be provided through following relation:

$$
z^{0}=T^{-1} x^{0}
$$

$x^{0}$ is the initial condition vector of the identified state space model. It is also provided by SSI algorithms.

Some investigators [24] discuss another kind of participation called State in Mode participation factor. In most of literatures state in mode and mode in state participation factors are considered the same as each other and they have been used interchangeably. However,there is a discussion on some differences in [24]. The following proposition challenges the definition of state in mode participation factor. 
Proposition 1. "State in Mode Participation" is a meaning-less expression.

Proof: A system includes some physical elements (such as resistors, capacitors and inductors in electrical systems or dampers, springs and masses in mechanical systems). Each element has a value and a role in topology and configuration of system. Configuration and topology of system provides the system with unique set of differential equations which we call it mathematical model.

A presentation of system mathematical model is State Space Structure which has a system matrix called $A$ which is affected by basic elements and configuration of system. Systems modes are one of the mathematical properties of $A$, therefore system modes only rely on system elements and system configuration. System states don't affect its modes so there is no state in mode participation and system modes are not affected by its states.

Example 2. For instance, one can provide the series $R L C$ circuit with the following state space model:

$$
\left[\begin{array}{c}
\dot{x}_{1} \\
\dot{x}_{2}
\end{array}\right]=\left[\begin{array}{cc}
\lambda_{1} & 0 \\
0 & \lambda_{2}
\end{array}\right]\left[\begin{array}{l}
x_{1} \\
x_{2}
\end{array}\right]+\left[\begin{array}{c}
1 \\
1
\end{array}\right] v_{i}, \quad v_{c}=\left[\begin{array}{ll}
\frac{\lambda_{1} \lambda_{2}}{\left(\lambda_{1}-\lambda_{2}\right)} & \frac{-\lambda_{1} \lambda_{2}}{\left(\lambda_{1}-\lambda_{2}\right)}
\end{array}\right]\left[\begin{array}{l}
x_{1} \\
x_{2}
\end{array}\right]
$$

where

$$
\lambda_{1,2}=-\xi \omega_{n} \pm j \omega_{n} \sqrt{1-\xi^{2}}, \omega_{n}=1 / \sqrt{L C}, \quad \xi=(R / 2) / \sqrt{L / C}
$$

Therefore, $\lambda_{1,2}$ rely only on $L, C$ and $R$ and the topology of circuit and the states depend on system modes $\lambda_{1,2}$. Thus, there is no participation of states in modes, and the expression state in mode participation is meaningless.

\section{$5 \quad$ Test Cases and Simulations}

Identification process should be provided with sampled input/output signals. Therefore, Computer simulations of the following test case power systems have been conducted using MATLAB/SIMULINK installed on a computer with $2.4 \mathrm{GHz}$ CPU and 4G RAM.

We study the systems shown in Figure 1; A single machine connected to an infinite bus, and a four-machine two-area system. Since we may extract the small signal properties of generator angle and speed, it is recommended to use mechanical torque as input and rotor speed and/or its angle as output signals. Torque and power are the same in per-unit system. Therefore, we use mechanical power as input signal. In order to have enough persistency of excitation in input signals, we added a white noise to input signals. To provide more realistic operating conditions, we added a white noise to output signals, as well. Effect of noises will be investigated later.

\subsection{Single Machine Three-Bus System}

Power system shown in the left side of Figure 1 is a three bus single machine power system with no control and exciter. The parameters of the system are those used in [23]. We are supposed to extract all small signal properties of system using SSI algorithms and the methods illustrated in previous sections.

We acquired 300 samples of input/output data through a 30 second simulation. Using the SSI algorithms presented in Table 1 , some linear models were identified and the results are presented in Table 2. It is clear that for investigating performance of noises, we cannot manipulate their averages since the operating point may vary. This is not applicable in this study. Therefore, each noise variance was altered separately in order to see its effect. In Table 2, It is expressed that an increase in input noise variance may lead to a better model from the view of FPE (Final Prediction Error) measure. However, we should be conservative when the estimation of 

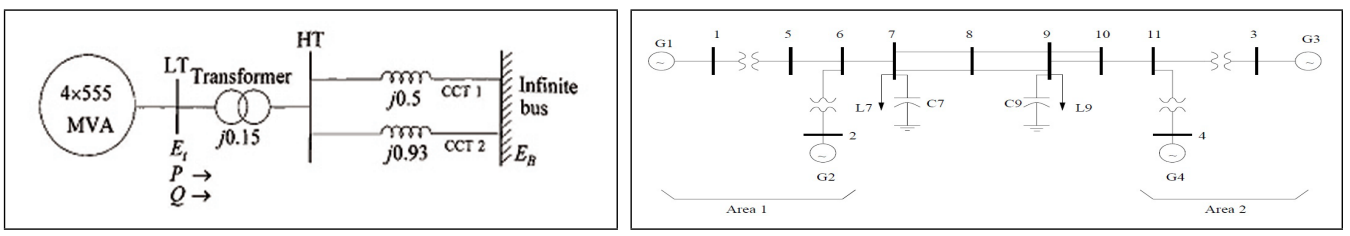

Figure 1: Left; Single machine three-bus power system. Right; Two-area four-machine power system.

\begin{tabular}{|c|c|c|c|c|c|c|c|c|}
\hline & $\sigma_{u}$ & $\sigma_{y}$ & $\lambda$ & $\xi$ & $\omega_{n}(H z)$ & \multicolumn{2}{|c|}{$|P|$} & FPE \\
\hline \multirow{2}{*}{ CM } & \multirow[t]{2}{*}{ - } & \multirow[t]{2}{*}{ - } & \multirow{2}{*}{$-0.714 \pm j 6.35$} & \multirow{2}{*}{0.112} & \multirow{2}{*}{1.0165} & 0.50 & 0.503 & \multirow[t]{2}{*}{-} \\
\hline & & & & & & 0.50 & 0.503 & \\
\hline \multirow{2}{*}{ SSIM01 } & \multirow{2}{*}{0.0001} & \multirow{2}{*}{0} & \multirow{2}{*}{$-0.7975 \pm j 6.2443$} & \multirow{2}{*}{0.1276} & \multirow{2}{*}{0.9988} & 0.503 & 0.5037 & \multirow{2}{*}{$7.4 \mathrm{e}-5$} \\
\hline & & & & & & 0.503 & 0.5037 & \\
\hline \multirow{2}{*}{ SSIM02 } & \multirow{2}{*}{0.001} & \multirow{2}{*}{0} & \multirow{2}{*}{$-0.7192 \pm j 6.3203$} & \multirow{2}{*}{0.1139} & \multirow{2}{*}{1.0105} & 0.502 & 0.5028 & \multirow{2}{*}{$8.5 \mathrm{e}-5$} \\
\hline & & & & & & 0.502 & 0.5028 & \\
\hline \multirow{2}{*}{ SSIM03 } & \multirow{2}{*}{0.01} & \multirow{2}{*}{0} & \multirow{2}{*}{$-0.7727 \pm j 6.1300$} & \multirow{2}{*}{0.1253} & \multirow{2}{*}{0.9825} & 0.503 & 0.5033 & \multirow{2}{*}{$11 e-5$} \\
\hline & & & & & & 0.503 & 0.5033 & \\
\hline \multirow{2}{*}{ SSIM04 } & \multirow{2}{*}{0.001} & \multirow{2}{*}{0.01} & \multirow{2}{*}{$-0.7192 \pm j 6.3203$} & \multirow{2}{*}{0.1139} & \multirow{2}{*}{1.0105} & 0.502 & 0.5028 & \multirow{2}{*}{0.59} \\
\hline & & & & & & 0.502 & 0.5028 & \\
\hline \multirow{2}{*}{ SSIM05 } & \multirow{2}{*}{0.001} & 0.01 & $-07102+i 63203$ & 01130 & 10105 & 0.502 & 0.5028 & 060 \\
\hline & & & $-0.1192 \pm j 0.3203$ & 0.1139 & 1.0100 & 0.502 & 0.5028 & 0.00 \\
\hline
\end{tabular}

Table 2: Small Signal Analysis of Single Machine Power System Using SSI Algorithms; abriviations are: Classical Model (CM), Subspace System Identified Model (SSIM), Participation Matrix $(\mathrm{P})$, variance of input noise $\left(\sigma_{u}\right)$, variance of output noise $\left(\sigma_{y}\right)$, eigenvalue $(\lambda)$, damping factor $(\xi)$, natural frequency $\left(\omega_{n}\right)$, and Final Prediction Error (FPE).

small signal properties is under consideration. Actually, a large increase in input noise variance may alter the operating point or its absorption area. This may lead to instability. In Table 2, if we compare SSIM4 and SSIM5 with SSIM2, we can see that output noise has no effect on subspace system identification. Actually, additive output noise does not have considerable effects on the SSI identification, since SSI algorithms use robust linear algebra tools. It is noticeable that the Left eigenvector of a wide matrix are not considerably sensitive to additive white noise, [2]. Therefore, the identification is not sensitive to additive output noise. What is more, the identification process has no effect on normal operating conditions of power system, since the applied input noise is too weak.

\subsection{Two-Area Four-Machine System}

The power system introduced in [25] is used as the second case study (see the right side of Figure 1). This power system has four generators and two fully symmetrical areas linked together by a weak line. It was specifically designed [23] to study low frequency electromechanical oscillations in large interconnected power systems. Despite its small size, it can thoroughly mimic the behavior of typical systems in actual operation.

In the case of no PSS (Power System Stabilizer), when the measured signals are differential speeds and differential angles of all generators, the CVA algorithm detected 10 modes shown in Figure 2. As illustrated in Figure 2, there are 8 oscillatory modes, and two non-oscillatory modes. There are three zero modes, since there is no infinite bus as a reference for rotor angles. 


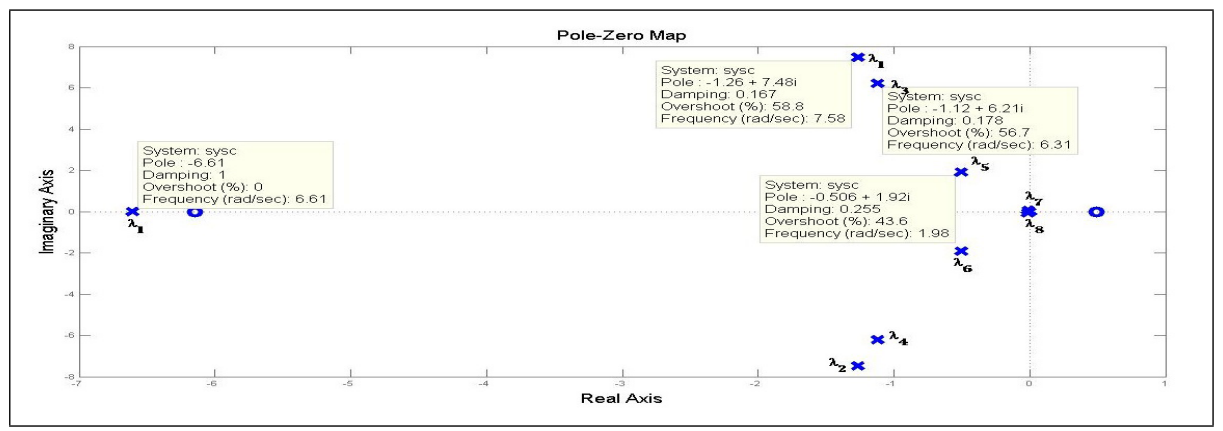

Figure 2: Pole-zero map of the identified model foe two-area system (No PSS) using CVA.
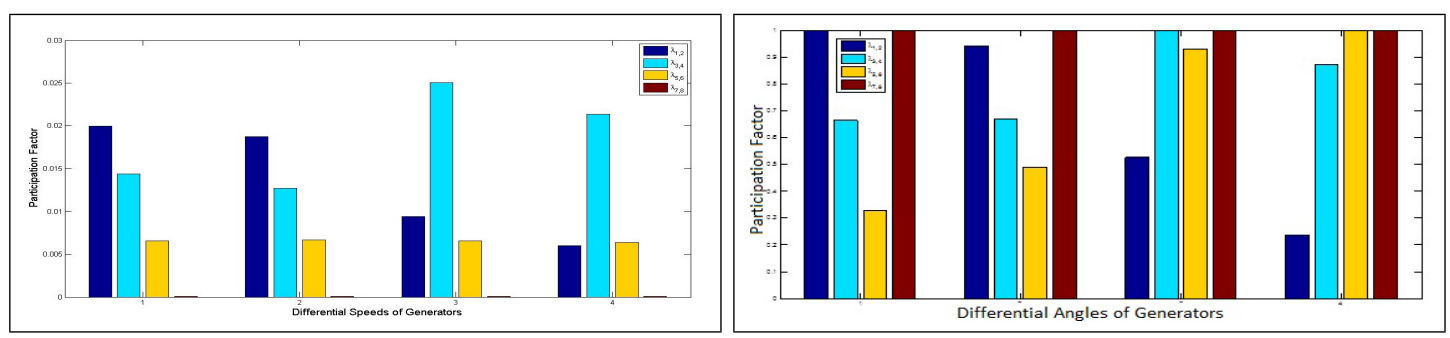

Figure 3: Left: Normalized participation factors of modes in differential speeds of generators. Right: Normalized participation factors of modes in differential Angles of generators. (No PSS, measurements are differential Angles and differential speeds).

Moreover, the speed governors have not been modeled [23], ( $\lambda_{7,8}$ and a real pole in the origin).

In order to distinguish between local and inter-area modes, the participation factors of modes are illustrated in Figure 1. Participation factors were calculated using the approach presented in previous section. Table 3 illustrates the small signal properties of two-area system extracted from Figure 2 and Figure 1.

In order to damp oscillatory modes, different power system stabilizers have been designed and applied to two-area power system. Performances of PSS have been investigated using SSI algorithms and the method mentioned in previous section. Table 4 summarizes the damping performance of Multi-Band (MB) PSS, $\Delta \omega$ PSS and $\Delta P a$ PSS [26] when applied to two-area power system. All PSSs perform very well. They stabilize the naturally unstable system. However, it is clear that the MB-PSS is superior to the other two PSSs, since it provides significantly more damping for all modes. The results presented here using identification method, are identical to those analytically achieved in [26].

\begin{tabular}{|c|c|c|c|}
\hline Mode & $\omega_{d}(\mathrm{~Hz})$ & $\xi(\%)$ & Participated States \\
\hline$\lambda_{1,2}$ & 1.1904 & 16.70 & $\delta_{1}, \delta_{2}$ (Local, Area 1) \\
\hline$\lambda_{3,4}$ & 0.9871 & 17.84 & $\delta_{3}, \delta_{4}, \omega_{3}, \omega_{4}$ (Local, Area 2) \\
\hline$\lambda_{5,6}$ & 0.3061 & 24.35 & $\delta_{3}, \delta_{4}, \omega_{1}, \omega_{2}, \omega_{3}, \omega_{4}$ (Inter-area) \\
\hline$\lambda_{7,8}$ & 0.0095 & 20.78 & $\delta_{1}, \delta_{2}, \delta_{3}, \delta_{4}, \omega_{1}, \omega_{2}, \omega_{3}, \omega_{4}$ (Inter-area) \\
\hline
\end{tabular}

Table 3: Small Signal Properties of Two Area Test System (No PSS). 


\begin{tabular}{|c|c|c|c|}
\hline PSS & $\omega_{d}(\mathrm{~Hz})$ & $\xi(\%)$ & Mode Type \\
\hline No PSS & 1.1904 & 16.70 & Local \\
& 0.9871 & 17.84 & Local \\
& 0.3061 & 24.35 & Inter-area \\
\hline MB PSS & 0.3942 & 51.91 & Inter-area \\
\hline$\Delta \omega$ PSS & 0.7115 & 47.71 & Inter-area \\
& 0.1113 & 67.82 & Controller \\
\hline Pa PSS & 0.4655 & 43.02 & Inter-area \\
\hline
\end{tabular}

Table 4: Investigating the effects of PSS on Oscillatory modes of two-area system using SSI.

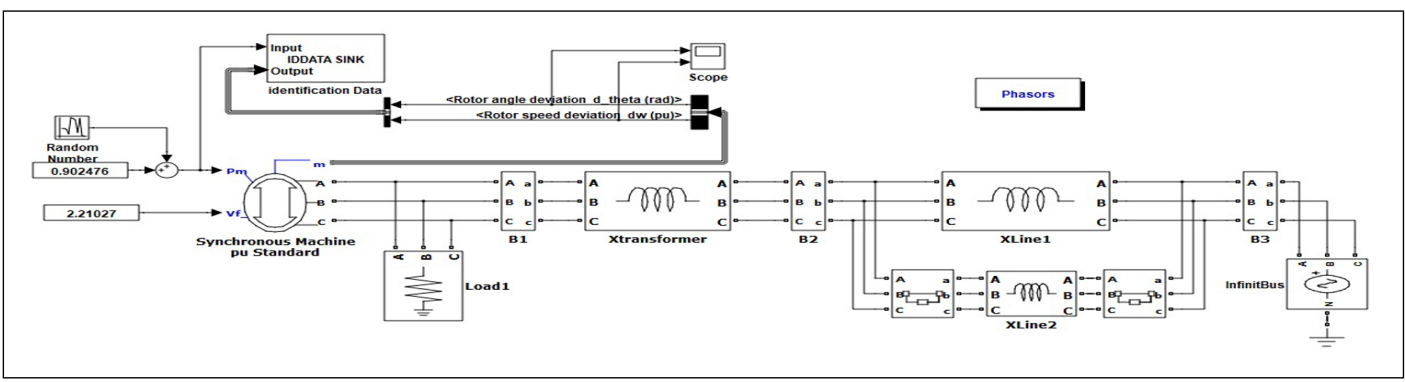

Figure 4: Monitoring of single machine three-bus system

\section{On-line Monitoring of Power System Using SSI}

On-line monitoring of power system is an open area which attracts tremendous consideration of investigators. Using analytic method for on-line monitoring may lead in calculation problems and the results are not also trustfully. Since the identification process uses sampled signals of power system, there would not be any gap between identification results and power system behavior. Therefore, the results are trustworthy in the case of power system monitoring. SSI algorithms can easily be applied to on-line power system monitoring. They can provide a state space model which is most suitable for on-line monitoring.

A single machine three-bus power system with two transmission lines is used to investigate on-line monitoring of power systems (Figure 2). Some computer simulations were conducted for 60 second. In the first 30 seconds, both lines are in operating condition. As a result of a fault, for the second 30 seconds, line 2 is out of work. Some state space models were identified in every 20 seconds using SSI algorithms. There would be three models and the operating point will change for the second model, so one can call it as an operating point transient model.

Three identified model and their properties are available in Table 5. SSIM6 represents the system performance in the initial operating point before the fault. SSIM8 represents system performance after breaking out of second line. SSIM7 is a middle model between SSIM6 and SSIM8.

As it can be seen from the column of modes column in Table 5 , modes has moved slightly to the right and as a result stability of system has reduced. It implies that the operating point should be re-tuned in order to recover more stable operating conditions.

Damping frequency and also damping factor has reduced. It implies the need for some control in order to provide power system with more suitable synchronizing and damping factors.

It is considerable that the whole monitoring process expresses a little movement of eigenvalues toward the right half plane. Moreover, there is a decrease in damping frequency. Therefore, a control should be applied to mechanical torque and field voltage, because it is predictable that a variation in operating conditions of power system may lead to instability and oscillatory behavior. 


\begin{tabular}{|c|c|c|c|c|c|}
\hline Model & $\lambda$ & $\omega_{d}(H z)$ & $\xi$ & & \\
\hline \multirow{2}{*}{ SSIM6 } & \multirow{2}{*}{$-0.6010 \pm j 8.0143$} & \multirow{2}{*}{1.2755} & \multirow{2}{*}{0.0748} & 0.5016 & 0.5016 \\
\hline & & & & 0.5016 & 0.5016 \\
\hline \multirow{2}{*}{ SSIM7 } & \multirow{2}{*}{$-0.5269 \pm j 7.0677$} & \multirow{2}{*}{1.1249} & \multirow{2}{*}{0.0743} & 0.5012 & 0.5012 \\
\hline & & & & 0.5012 & 0.5012 \\
\hline \multirow{2}{*}{ SSIM8 } & \multirow{2}{*}{$-0.4810 \pm j 6.9452$} & \multirow{2}{*}{1.1054} & \multirow{2}{*}{0.0691} & 0.5018 & 0.5018 \\
\hline & & & & 0.4775 & 0.4775 \\
\hline
\end{tabular}

Table 5: Subspace System Identification Models (SSIM) identified during Online Monitoring.

\section{$7 \quad$ Effect of Different SSI Algorithms on Small Signal Analysis of Power Systems}

There are several kinds of subspace system identification algorithms. They may all use the same mathematical tools. But they are different in applying tools to measurement data. Therefore, SSI algorithms may show slightly different results for the same input/output set of data. There are three main algorithms which are usually used in the papers discussing power system analysis; MOESP (Multi-variable Output-Error State Space), N4SID (Numerical State Space Subspace System IDentification) and CVA (Canonical Variate Analysis). We are supposed to compare performance of such algorithms when they are used for small signal analysis of power systems in this section.

Figure 1 shows the system under study. Small signal analysis of mentioned power system was conducted using four models and different SSI algorithms. The models were investigated; 1) Classical model of 3-bus single machine power system which has no control and the effect of field is ignored. 2) Single machine power system model containing effects of field voltage. 3) Auto-voltage regulated (AVR) power system. 4) Single machine power system with AVR and power system stabilizer (PSS). In each case, $K_{D}$ were calculated using the system parameters available in [23].

Table 6 contains results of small signal analysis for several models of single machine power system using two different SSI algorithms. Identification results are almost the same for two SSI algorithms. They are slightly different in estimation of non-oscillatory modes. Table 6 also expresses that SSI algorithms are not capable of identifying non-dominant modes. That is not a draw-back, since non-dominant modes are not so important for system analysis. As a whole, one can use subspace system identification algorithms for sure, when it is needed to estimate most effective modes of power system. Therefore, Subspace system identification algorithms are convenient solution for extracting small signal stability properties of a power system.

\section{Conclusions and Future Works}

The paper discusses pitfalls of small signal analysis of power systems when using analytic methods. Analytic methods use basic theories and the provided parameters in order to extract operating point and linearized model of power system. In this paper, it is expressed that we can avoid such boring, time consuming and cost effective stages, using system identification methods. The most proper method for power system small signal analysis is then, application of subspace system identification (SSI) algorithms. SSI methods have many other advantages which were discussed in the paper. Extraction of modes and their participation factors are easy when using SSI methods but it needs some modification in SSI algorithms. In order to fulfill such requirements, some suggestions presented in this paper. Using SSI algorithms, some ideas 


\begin{tabular}{|c|c|c|c|c|}
\hline No. & & Classical & MOESP & CVA \\
\hline \multirow{3}{*}{1} & $\lambda$ & $0 \pm j 6.39$ & $0 \pm j 6.4158$ & $0 \pm j 6.4158$ \\
\hline & $\xi$ & 0.0000 & 0.0000 & 0.0000 \\
\hline & $\omega_{d}$ & $1.0200 \mathrm{~Hz}$ & $1.0211 \mathrm{~Hz}$ & $1.0211 \mathrm{~Hz}$ \\
\hline \multirow{3}{*}{2} & $\lambda$ & $\lambda_{1,2}=-0.11 \pm j 6.41$ & $\lambda_{1,2}=-0.1095 \pm j 6.4114$ & $\begin{array}{l}\lambda_{1,2}=-0.1095 \pm j 6.4114 \\
\lambda_{2}=-0.2038\end{array}$ \\
\hline & $\xi$ & 0.0170 & 0.0171 & 0.0171 \\
\hline & $\omega_{d}$ & $1.0700 \mathrm{~Hz}$ & $1.0204 \mathrm{~Hz}$ & $1.0204 \mathrm{~Hz}$ \\
\hline \multirow{5}{*}{3} & & $\lambda_{1,2}=+0.5040 \pm j 7.2300$ & $\lambda_{1,2}=+0.5045 \pm j 7.2321$ & $\lambda_{1,2}=+0.5045 \pm j 7.2321$ \\
\hline & $\lambda$ & $\lambda_{3}=-20.202$ & $\lambda_{3}=-0.0015$ & $\lambda_{3}=-0.0310$ \\
\hline & & $\lambda_{4}=-31.230$ & $\lambda_{4}=-15.1521$ & $\lambda_{4}=-15.1174$ \\
\hline & $\xi$ & -0.0700 & -0.0696 & -0.0696 \\
\hline & $\omega_{d}$ & $1.1500 \mathrm{~Hz}$ & $1.1510 \mathrm{~Hz}$ & $1.1510 \mathrm{~Hz}$ \\
\hline \multirow{8}{*}{4} & & $\lambda_{1}=-0.7390$ & $\lambda_{1}=0.0000$ & $\lambda_{1}=0.0000$ \\
\hline & 1 & $\lambda_{2,3}=-1.005 \pm j 6.607$ & $\lambda_{2}=-4.5770$ & $\lambda_{2}=-4.6266$ \\
\hline & $\lambda$ & $\lambda_{4,5}=-19.797 \pm j 12.822$ & $\lambda_{3,4}=-1.0357 \pm j 6.6150$ & $\lambda_{3,4}=-0.9946 \pm j 6.5951$ \\
\hline & & $\lambda_{6}=-39.097$ & $\lambda_{5,6}=-16.857 \pm j 16.288$ & $\lambda_{5,6}=-16.906 \pm j 16.265$ \\
\hline & $\varepsilon$ & $\xi_{2,3}=0.15$ & $\xi_{3,4}=0.1547$ & $\xi_{3,4}=0.1491$ \\
\hline & $\xi$ & $\xi_{4,5}=0.84$ & $\xi_{5,6}=0.7191$ & $\xi_{5,6}=0.7204$ \\
\hline & & $\omega_{d 2,3}=1.05 \mathrm{~Hz}$ & $\omega_{d 2,3}=1.0528 \mathrm{~Hz}$ & $\omega_{d 2,3}=1.0496 \mathrm{~Hz}$ \\
\hline & $\omega_{d}$ & $\omega_{d} 4,5=2.04 H z$ & $\omega_{d 4,5}=2.5924 H z$ & $\omega_{d 4,5}=2.5886 \mathrm{~Hz}$ \\
\hline
\end{tabular}

Table 6: Small signal analysis of single machine power system using different types of subspace system identification algorithms. 1) Classical model (No field control, $\mathrm{K}_{D}=0$ ) . 2) System model containing effects of field voltage, $\left(\mathrm{K}_{D}=1.53\right)$. 3) Auto-voltage regulated (AVR) system, $\left.\left(K_{D}=-7.06\right) .4\right)$ System model with AVR and power system stabilizer (PSS), $\left(K_{D}=14.08\right)$ 
such as power system monitoring can be conducted in a more convenient and more realistic way. This point is discussed in several parts of paper while applying SSI algorithms to different power systems.

Future works may aim at application of subspace system identification methods to monitor modes, stability measures and estimation of damping and synchronous factors of power system.

\section{Bibliography}

[1] P. van Overschee and B. de Moor, A unifying theorem for three subspace system identification algorithms, Automatica, vol. 31, pp. 1853-1864, 1995

[2] T. Katayama, Subspace Methods for System Identification: Springer, 2005

[3] S. J. Qin, An Overview of Subspace Identification, Elsevier, Computer $\&$ Chemical Engineering, vol. 30, pp. 1502-1513, 2006

[4] ] P. Van Overschee and B. De Moor, "N4SID: Subspace algorithms for the identification of combined deterministic-stochastic systems, Automatica, vol. 30, pp. 75-93, 1994

[5] M. Verhaegen and P. Dewilde, Subspace model identification Part 1. The output-error statespace model identification class of algorithms, International Journal of Control, vol. 56, pp. 1187-1210, 1992/10/01 1992

[6] M. Verhaegen and P. Dewilde, Subspace model identification Part 2. Analysis of the elementary output-error state-space model identification algorithm, International Journal of Control, vol. 56, pp. 1211-1241, 1992/10/01 1992

[7] M. Viberg, Subspace-based methods for the identification of linear time-invariant systems, Automatica, vol. 31, pp. 1835-1851, 1995

[8] M. Verhaegen, Identification of the deterministic part of MIMO state space models given in innovations form from input-output data, Automatica, vol. 30, pp. 61-74, 1994

[9] W. E. Larimore, "Canonical variate analysis in identification, filtering, and adaptive control, in Decision and Control, Proceedings of the 29th IEEE Conference on, 1990, pp. 596-604

[10] S. J. Qin, An overview of subspace identification, Computers \& Chemical Engineering, vol. 30, pp. 1502-1513, 2006

[11] G. T. I. Kamwa, L. Gerin-Lajoe, Low Order Black-Box Models for Control System Design Large Power Systems, IEEE Transaction on Power Systems, Vol. 11, No. 1, February 1996

[12] S. M. I. H. Akcay, B. Ninness, Subspace-Based Identification of Power Transformer Models from Frequency Responce Data, IEEE Transaction on Instrumentation and Measurement, Vol. 48, No. 3, June 1999

[13] H. A. T. McKelvey, Lennart Ljung, Subspace-Based Multivariable System Identification from Frequency Responce Data, IEEE Transaction on Automatic Control, Vol. 41, No. 7, July 1996

[14] S. M. I. H. Akcay, B. Ninness, Identification of Power Transformer Models from Frequency Responce data: A Case Study. Elsevier, Signal Processing, 68, pp 307-315, 1998 
[15] O. P. M. M. Karrari, Identification of Heffron-Phillips Model Parameters for Synchronous Gemerators Using Online Measurment, IEEE Proceeding of Generator, Transmition, Distribution, Vol. 151, No. 3, May 2004.

[16] D. W. O. P. M. M. Soliman, Identification of Heffron-Phillips Model Parameters for Synchronous Generators Operating in Closed Loop, IEEE Generation, Transmition and Distribution, 2(4):530-541, 2008

[17] O. P. M. Bin Wu. Multivariable Adaptive Control of Synchronous Machines in a Multimachine Power System. IEEE Transaction on Power Systems, Vol. 21, No. 4, November 2006

[18] J. W. P. Ning Zhou, J. F. Hauer, Initial Results in Power System identification From Injected Probing Signals Using a Subspace Method, IEEE Transaction on Power Systems, Vol. 21, No. 3, August 2006

[19] C. C. H. Ghasemi, A. Moshref, Oscillatory Stability Limit Prediction Using Stochastic Subspace Identification, IEEE Transaction on Power Systems, 21(2):736-745, 2006

[20] L. G.-L. I. Kamwa, G. Trudel, Multi-Loop Power System Stablizers Using Wide-Area Synchronous Phasor Measurements, Proceedings of the American Control Conference, Philadelphia, Pennsylvania, June 1998

[21] D. Y. G Cai, Y. Jiao, Ch. Shao, Power System Oscillation Mode Analysis and Parameter Determination of PSS Based on stochastic Subspace Identification, Asia-Pacific Power and Energy Engineering Conference (APPEEC), pp. 1-6, March 2009

[22] P. Overschee and B. L. R. Moor, Subspace identification for linear systems: theory, implementation, applications: Kluwer Academic Publishers, 1996

[23] PP. Kundur and N. J. Balu, Power System Stability and Control: IEEE, 1998

[24] M. A. H. Wael A. Hashlamoun, and Eyad H. Abed, New Results on Modal Participation Factors: Revealing a Previously Unknown Dichotomy. IEEE Transactions of Automatic Control, Vol. 54, No. 7, July 2009

[25] J. J. Sanchez-Gasca, V. Vittal, M. J. Gibbard, A. R. Messina, D. J. Vowles, S. Liu, and U. D. Annakkage, Inclusion of higher order terms for small-signal (modal) analysis: committee report-task force on assessing the need to include higher order terms for small-signal (modal) analysis, Power Systems, IEEE Transactions on, 20(4):1886 - 1904, 2005

[26] R. Klein, Moorty and Kundur. Analytical investigation of factors influencing PSS performance, IEEE Trans. on EC, 7(3):382-390, 1992 